Max-Planck-Institut für demografische Forschung

Max Planck Institute for Demographic Research

Doberaner Strasse 114 - D-18057 Rostock · GERMANY

Tel +49 (0) 3812081 - 0; Fax +49 (0) 3812081 - 202;

http://www.demogr.mpg.de

MPIDR WORKING PAPER WP 2001-007

MARCH 2001

\title{
Examining Structural Shifts in Mortality Using the Lee-Carter Method
}

Lawrence R. Carter (lcarter@oregon.uoregon.edu)

Alexia Prskawetz (fuernkranz@demogr.mpg.de)

(C) Copyright is held by the authors.

Working papers of the Max Planck Institute for Demographic Research receive only limited review. Views or opinions expressed in working papers are attributable to the authors and do not necessarily reflect those of the Institute. 


\title{
Examining Structural Shifts in Mortality Using the Lee-Carter Method ${ }^{1}$
}

\author{
Lawrence R. Carter \\ Department of Sociology \\ University of Oregon \\ Eugene OR 97403 \\ USA \\ and \\ Alexia Prskawetz ${ }^{2}$ \\ Max Planck Institute for Demographic Research \\ 18057 Rostock \\ Germany
}

\begin{abstract}
We present an extension of the Lee-Carter method of modeling mortality to examine structural shifts in trajectories of mortality. Austrian data consisting of 53 years of single-age mortality rates are subdivided into 3024 -year submatrices. Using singular value decomposition, the submatrices are decomposed into three component submatrices: 1) the multiple realizations of the index of mortality to which each respective age-specific death rate is linearly related; 2 ) the average shape across age of the log of mortality schedules; 3 ) the sensitivity of the log of mortality at each age to variations in the elements of the index of mortality. We refer to these latter submatrices to locate structural changes in mortality patterns. A comparison of the observed and estimated life expectancy indicates that the extended Lee-Carter method is superior to the original Lee-Carter method, particularly so for life expectancies at higher ages. We conclude by projecting life expectancy up to 2050 , applying the Lee-Carter method to the whole time series (1947-1999) and comparing it to an application of the Lee-Carter method to the latest subsample (1976-1999).
\end{abstract}

\section{INTRODUCTION}

The Lee-Carter method (Lee and Carter 1992) of modeling mortality allows one to apply standard time series methods to forecast mortality. Because this method is computationally simple to apply, its use has become very popular in the course of the last decade for modeling and forecasting mortality patterns (Carter and Lee 1992, Lee and Nault 1993, Tuljapurkar et al. 2000, Wilmoth 1998).

However, several authors (e.g. Lee and Miller 2000) have recently noted that the assumption of a constant age shape of mortality - which is implicit in the original LeeCarter model - may be too rigid. As cited in Lee and Miller (2000), Horiuchi and Wilmoth (1995) present evidence for several countries that mortality has been declining more rapidly at higher ages than at lower ages during recent decades. In

\footnotetext{
${ }^{1}$ We would like to thank Alexander Hanika from Statistics Austria for providing the data.

${ }^{2}$ The views expressed in this paper are the author's own views and do not necessarily represent those of the Max Planck Institute for Demographic Research.
} 
this paper we present an extension of the original Lee-Carter method that takes into account the changing age pattern of mortality. Our method can be applied to locate structural changes in the historical pattern of mortality that could ultimately be used to determine the time horizon of past mortality rates on which forecasts should be based.

For this heuristic examination, we first apply the Lee-Carter method to time series mortality rates by single years of age for Austria from 1947 to 1999. These data are ideal for this examination because they show what is possibly a structural shift in mortality beginning around 1968. We attempt to attribute this shift to significant gains in life expectancy, $e_{0}$, derived from apparent gains in the health of the older population from that time forward. We then apply the Lee-Carter method to successive subsamples of equal length from the original time series of mortality rates. A comparison of the resulting age shape of mortality for the whole time series with the subsequent subsamples proves that Horiuchi and Wilmoth's assertion is true for Austria as well. We demonstrate that the extended Lee-Carter method also improves the fit for the observed life expectancy and may essentially influence the predicted gains in life expectancy.

This paper is divided into several sections. In Section 2, we present some historical background on Austrian mortality patterns to establish the setting for our research. Section 3 describes the basic nonlinear demographic model of the Lee-Carter method and the procedures for decomposing this model. Section 4 extends the decomposition of the Lee-Carter model to include time-varying age and time patterns of mortality to explore the sensitivity of these components to structural shifts in the data. In Section 5 we evaluate the fit of the estimated life expectancy between the original and the extended Lee-Carter approach. Section 6 presents a forecast of life expectancy to 2050 based on the whole time series 1947-1999 (original Lee-Carter approach) which we contrast with a forecast based on the last subsample from 19761999. Finally, in Section 7, we summarize our research and offer avenues for advancing this research endeavor.

\section{BACKGROUND}

Along with many industrialized western countries, Austria has seen a steady decline in mortality since the late 1940s. But the rate of decline has not been monotonic as indicated by a closer investigation of the life expectancy at birth for males and females (Figure 1). After a sharp decline in mortality in the late 1940s and the 1950s, it seemed that this trend came to an end in the 1960s. However, Austria experienced along with many industrialized western countries a further significant rise in life expectancy during the 1970s and 1980s.

In the 1970s a third of the gains in additional years of life were attributable to the decline in infant mortality, while it is declines in mortality among the elderly that have shaped the overall mortality decline during the last two decades. Parallel to the increase in life expectancy, the major causes of death shifted from infectious diseases to chronic-degenerative diseases and neoplasm. The main causes of death are now diseases of the circulatory system, neoplasms, and diseases of the digestive system. Moreover, the risk of death from several of these diseases has shifted to older ages in the course of the last decade. Olshansky and Ault (1986) characterized 
this development as 'the stage of delayed degenerative diseases'. Evidence for a delay in deaths from specific causes of death in Austria (e.g. malignant neoplasm of the digestive organs and peritoneum, heart diseases without acute myocardial infarction, etc.) is summarized in Kytir and Prskawetz (1995).

Figure 1: Life expectancy at birth for female and male populations in Austria, 1947-1999.

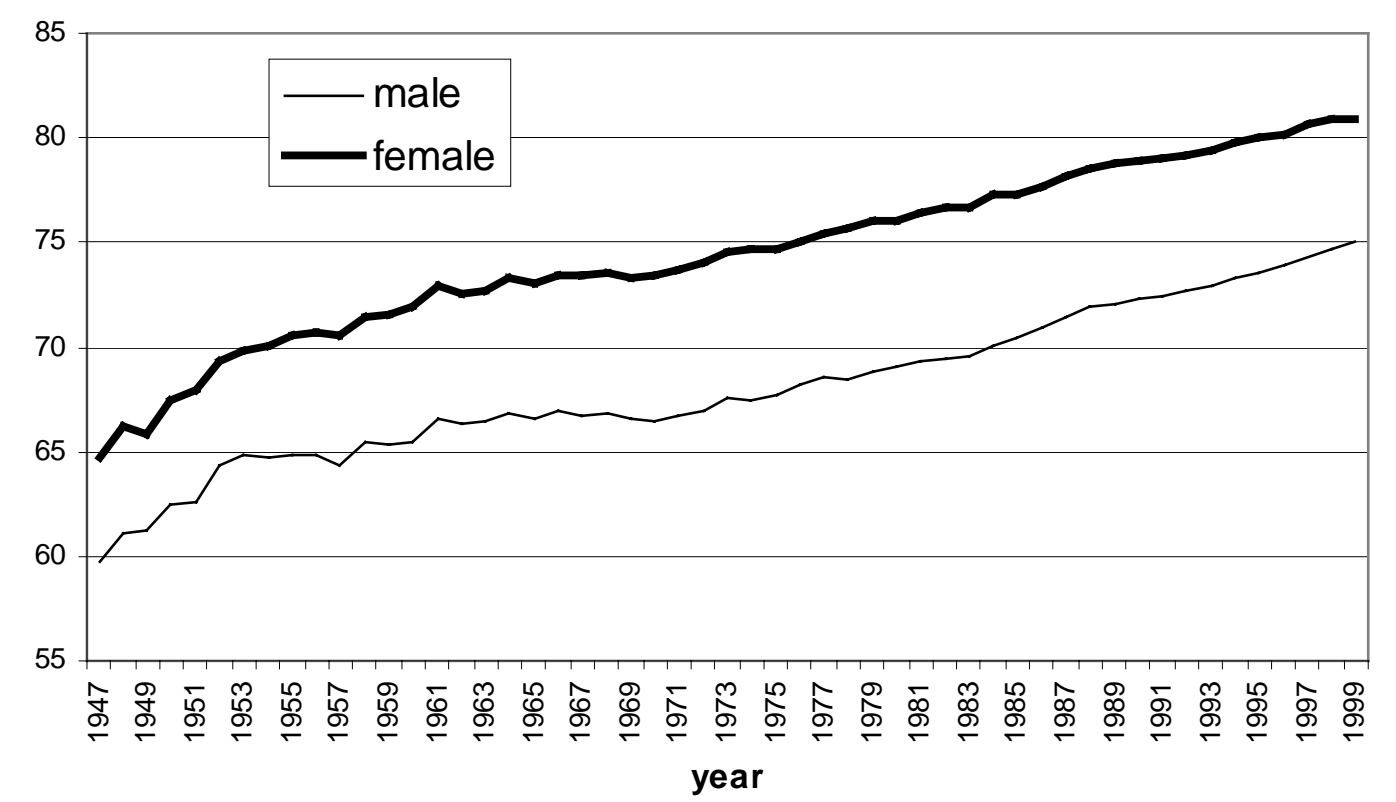

\section{A STOCHASTIC NONLINEAR DEMOGRAPHIC MODEL}

The components of the basic demographic model introduced by Lee and Carter (1992) are depicted in Figure 2 for males and females respectively. The model is designed to derive a single index of mortality $k_{t}$ (Figure 2.a) to which the log of each age-specific death rate is linearly related.

The model is of the form:

$$
\begin{aligned}
& m_{x, t}=\exp \left(a_{x}+k_{t} b_{x}+e_{x, t}\right) \text { or log transformed, } \\
& \ln \left(m_{x, t}\right)=a_{x}+k_{t} b_{x}+e_{x, t} \\
& \text { with } t=1947, \ldots, 1999 \text { and } x=0, \ldots, 95+
\end{aligned}
$$

Here $m_{x, t}$ is the central death rate for age $x$ at mortality level $k_{t}$, and $a_{x}$ (Figure $2 b$ ) is the average shape across age of the log of the mortality schedule. The variable $b_{x}$ (Figure $2 \mathrm{c}$ ) reflects the sensitivity of the log of mortality at age $x$ to variations in the parameter $k_{t}$. Linearization through the log transformation in (2) facilitates the extraction of $k_{t}$ using singular value decomposition (SVD).

For any value of $k_{t}$, the fitted model for (1) defines a set of central death rates which can be used to construct a life table. It is a mathematical implication of the model that, if $k_{t}$ changes linearly with time, then each age-specific rate changes at a 
constant exponential rate, where the constants vary by age. This relationship is evident in the transformation from equation (2) back to equation (1). The shape of the $b_{x}$ profile tells us which rates decline rapidly and which rates decline slowly over time. The error term, $e_{x, t}$, with mean zero and variance $s_{e}{ }^{2}$, reflects particular age-specific historical influences not captured in the model.

There are many ways to estimate $a_{x}, b_{x}$, and $k_{t}$. There have been three approaches that have been experimented with so far and all use singular value decomposition (SVD). Lee and Carter (1992) introduce a two stage process, while Wilmoth (1993) demonstrates a weighted SVD technique and a separate maximum likelihood method. There are only marginal differences among all four schemes. In this study we use Wilmoth's weighted SVD technique. ${ }^{3}$ Similar to Wilmoth (1993), we apply the normalizing constraint on $b_{x}: \sum_{x} b_{x}^{2}=1$ and the further necessary condition for full model identification $\sum_{t} k_{t}=0$.

As evident in Figure 2.a there is a clear downward trend in mortality across time, which resembles the non-monotonic pattern already observed in Figure 1: a sharp decline during the late 1940s and the 1950s, leveling off at the end of the 60s, and a further significant decline in the 1970s and 1980s. By definition, Figure 2.b plots the mean of the log mortality rates across time. The sensitivity of the log of mortality at age $x$ to variations in the elements of $k_{t}$ (Figure 2.c) is clear evidence of the advances in infant mortality during the early periods of the time series.

\section{INVESTIGATING FOR STRUCTURAL SHIFTS IN THE DATA}

In much of the early research on age-structured populations one assumed that these populations are stable or quasi-stable. Lee and Carter (1992) expand on the view of variable rates. Yet, they hold to the assumption of demographic stability. Once trajectories are established for age-specific death rates or life expectancies, it is assumed that they progress at fixed or uniformly changing rates toward some asymptotes. Here, however, we entertain the possibility of variances from stability, and that structural shifts or perturbations may have a significant impact on the outcome of a projection.

\section{4.a. Structural Shifts}

In the following we explore the stochastic behavior of the three components of (2). Thus, there are two age and time varying components, $a_{x, t}$ and $b_{x, t}$, and one time varying component, $\mathrm{k}_{\mathrm{t}}$. The decomposition of $\mathrm{m}_{\mathrm{x}, \mathrm{t}}$ is performed as described below. We look for what may be structural shifts in any of the components over time. Doing so allows us to come up with a stochastically richer rendition of the Lee-Carter model. We thus look for structural shifts, that is, discontinuities within the components. The discontinuities need not be abrupt, but they must be sufficiently abnormal to suggest that something of some behavioral significance must have occurred to destabilize the historical trajectory of the component. It is these discontinuities that we hope to

\footnotetext{
${ }^{3}$ We wish to thank Carolyn Hart and John R. Wilmoth, who made their Splus code of the weighted least squares estimate available to us.
} 
Figure 2: Decomposition of $\ln \left(m_{x, t}\right)$ for 1947-1999, female and male

Figure 2.a

time series of $k_{t}$

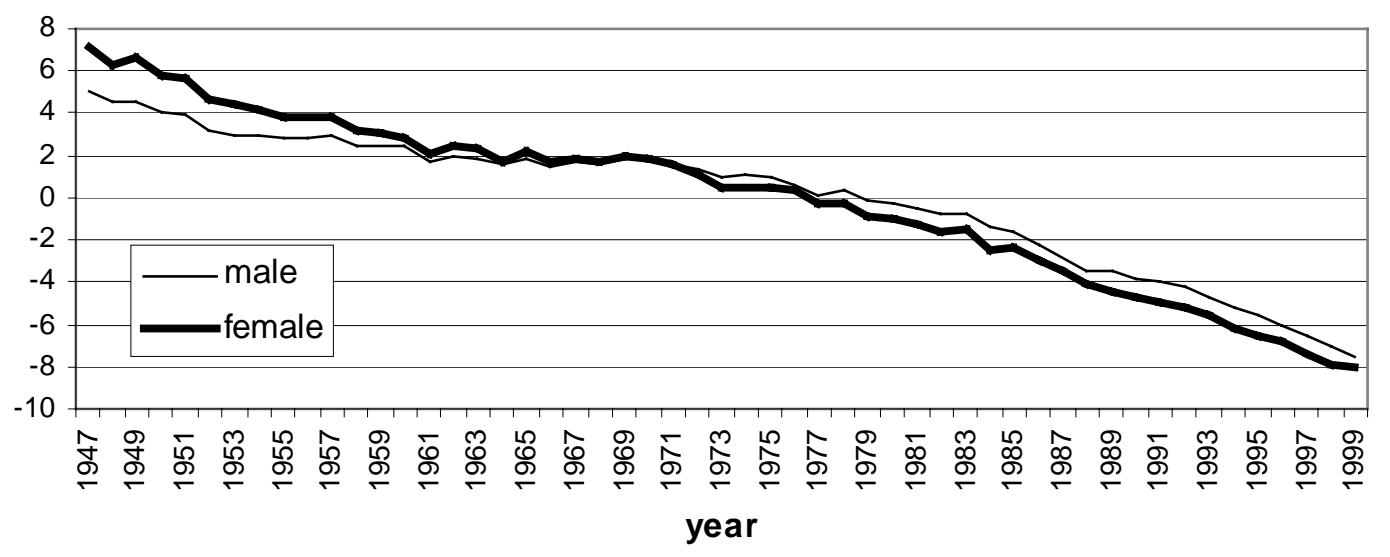

Figure 2.b

$a_{x^{-}}$schedule

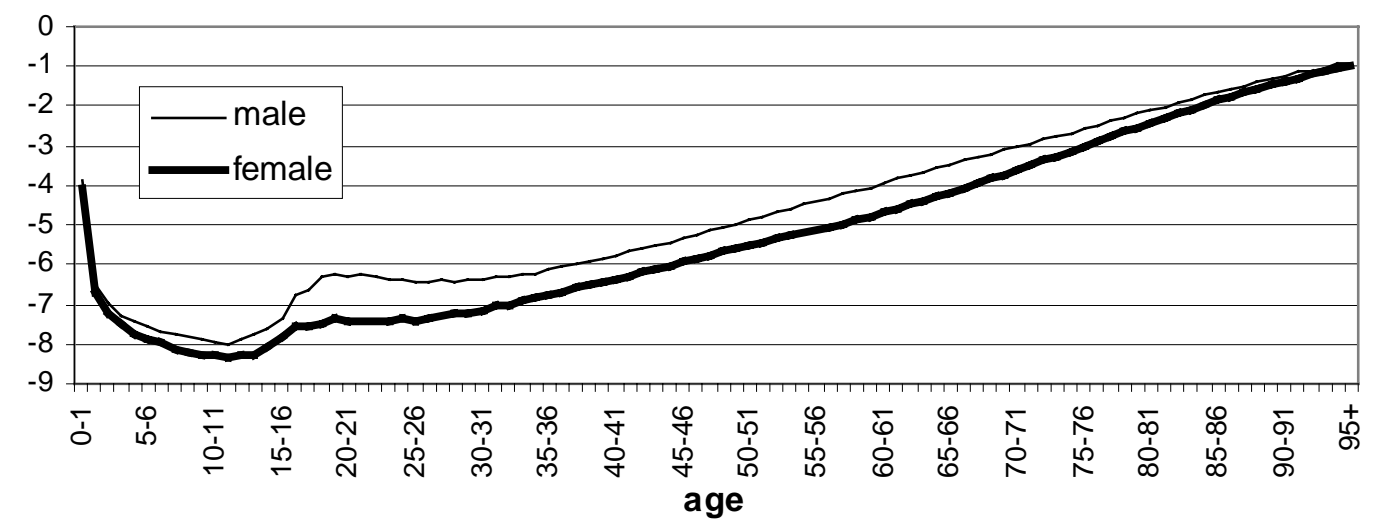

Figure 2.c $\quad b_{x}$-schedule

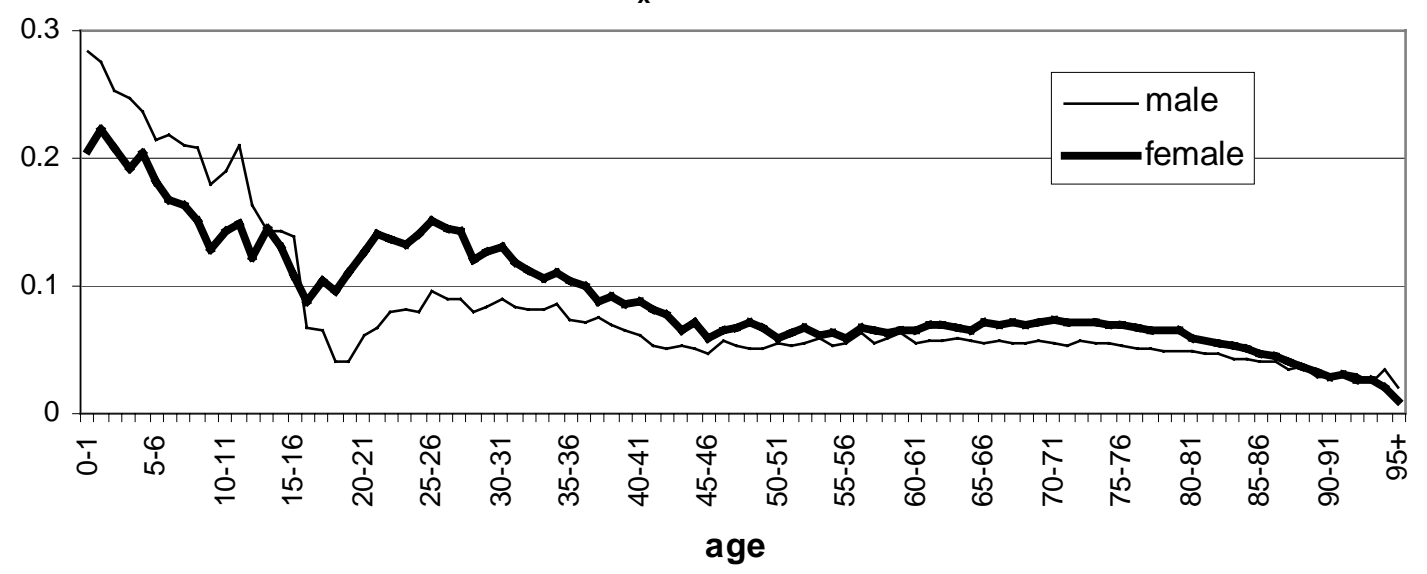


detect in this deeper analysis, which may ultimately provide more sensitive forecasts of mortality.

\section{4.b. Age and Time Varying Decomposition of $\mathrm{m}_{\mathrm{x}, \mathrm{t}}$ : the Extended Lee-Carter Model}

To extend the analysis so as to encompass the three time-varying components, equations (1) and (2) need to be reformulated. In matrix notation, they now become:

$$
\begin{aligned}
& M_{x, t_{s}}=\exp \left(A_{x, s}+K_{t_{s}} B_{x, s}+E_{x, t_{s}}\right) \text { and } \\
& \ln \left(M_{x, t_{s}}\right)=A_{x, s}+K_{t_{s}} B_{x, s}+E_{x, t_{s}} \\
& \text { with } s=1, \ldots, 30 ; t_{1}=1947-70, \ldots, t_{30}=1976-99 \text { and } x=0, \ldots, 95+
\end{aligned}
$$

where the components $t_{1}, \ldots, t_{30}$ represent blocks of 24 years sequentially sampled from the entire observed matrix of age-specific death rates $m_{x, t}$. Here, $K_{t_{s}} B_{x, s}$ is decomposed using SVD but in a manner different from that of Section 3. We sequentially sample 3024 -year submatrices of age-specific mortality rates from the total 53-year age-specific mortality rates for Austria. Each of the 30 submatrices as indicated by the subscript $s$ is decomposed so as to yield its own set of $a_{x}, b_{x}$, and $k_{t} s$. The elements of $B_{x, s}$ for selected values of $s$ (together with the $b_{x}$ schedule for the whole time series) are shown in Figures 3.a and 3.b for females and males respectively, and their corresponding contour plots (Andreev 1999) for all 30 subsamples $s$ are plotted in Figures $4 a$ and $4 b$. (Since the elements of $A_{x, s}$ and $K_{t_{s}}$ are much more stable across the subsamples, we concentrate exclusively on the schedule of the $B_{x, s}$ in this paper.)

From Figures 3.a. and 3.b. it is evident that the age pattern of mortality decline has changed considerably across our time of observation. A comparison of the $b_{x}$ schedule for the first (1947-1970) and the last (1976-1999) subsample shows a more stable pattern for the latter period for ages above 15 (cf. Lee and Miller 2000 for a similar conclusion). Applying the Lee-Carter method to the whole time series clearly averages out the distinct patterns at adult and higher ages exhibited in the two subsamples.

Even more intuitive is the representation of the whole $B_{x, s}$ schedule by means of contour plots as shown in Figures 4.a and 4.b. The cold (blue) areas indicate little change in mortality while the hot (orange and brown) regions indicate ages where mortality rates have declined most. These figures clearly illustrate the structural shifts in old age mortality since the 1970s, as shown by the shrinkage of the blue area in the upper age groups and the spread of the "hot" region into older ages. In the 1940s and up to the 1970s mortality improvements were mainly concentrated in the young and middle age groups, as is clearly visible in the hot regions around age 30 during these periods. The fact that we observe very high sensitivity at very low ages also in the latter periods might be due to the small numbers of people in these age groups, which means that even a modest mortality decline will have a pronounced effect. 
Figure 3.a: $b_{x}$ schedule for the entire time series 1947-1999 and two selected subsamples 1947-1970 and 1976-1999, female

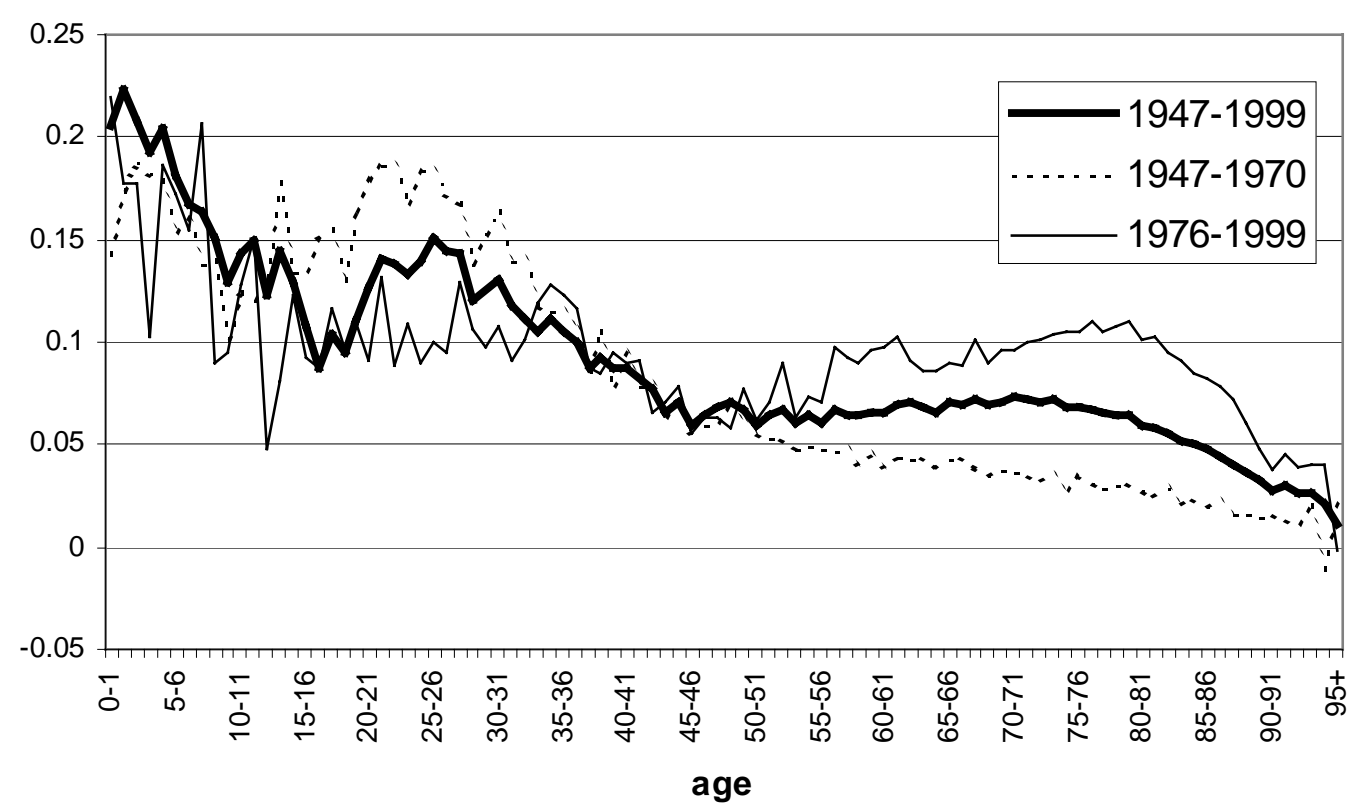

Figure 3.b: $b_{x}$ schedule for the entire time series 1947-1999 and two selected subsamples 1947-1970 and 1976-1999, male

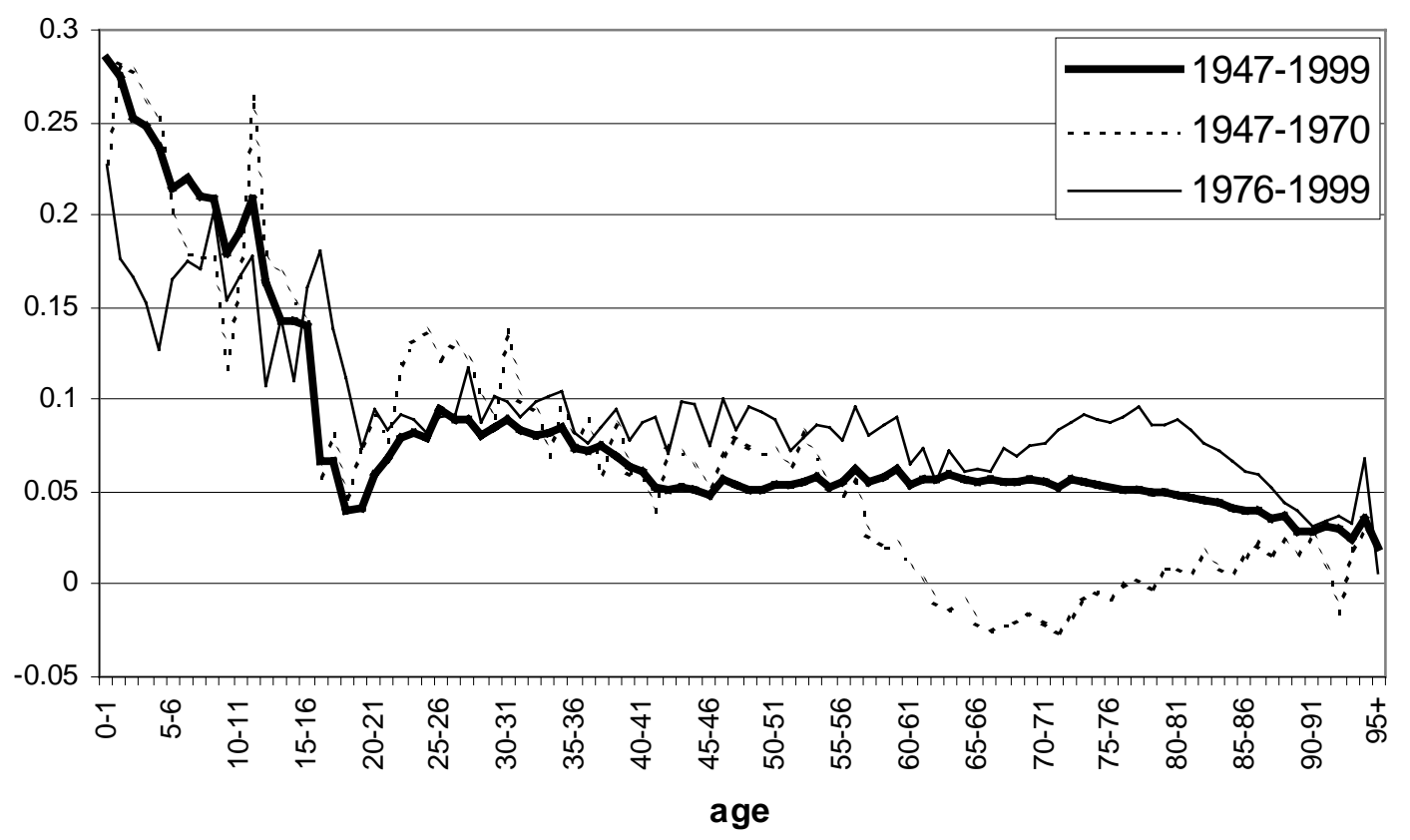


Figure 4a: Contour plot of $\mathrm{B}_{\mathrm{x}, \mathrm{s}}$ schedule, female

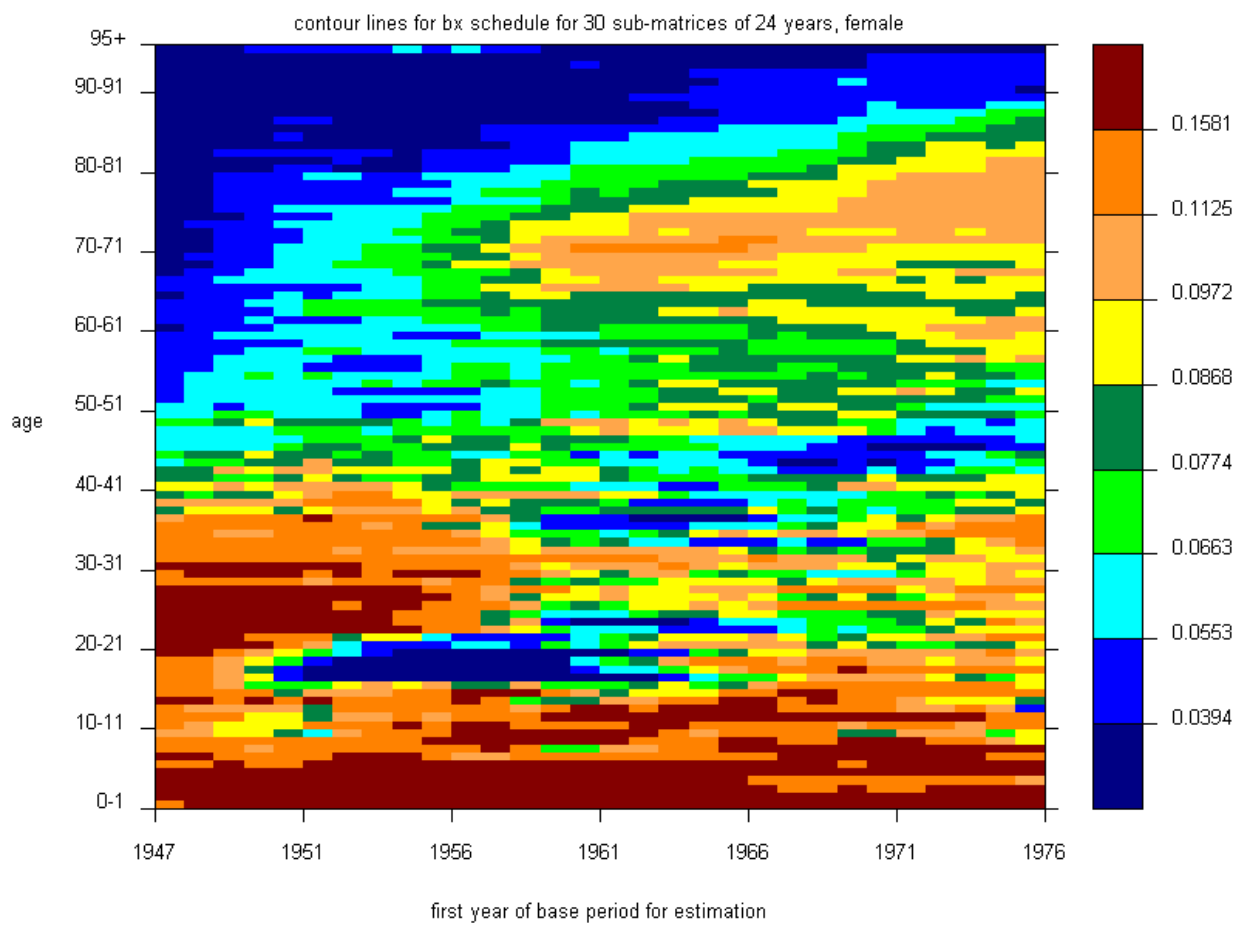

Figure 4b: Contour plot of $B_{x, s}$ schedule, male

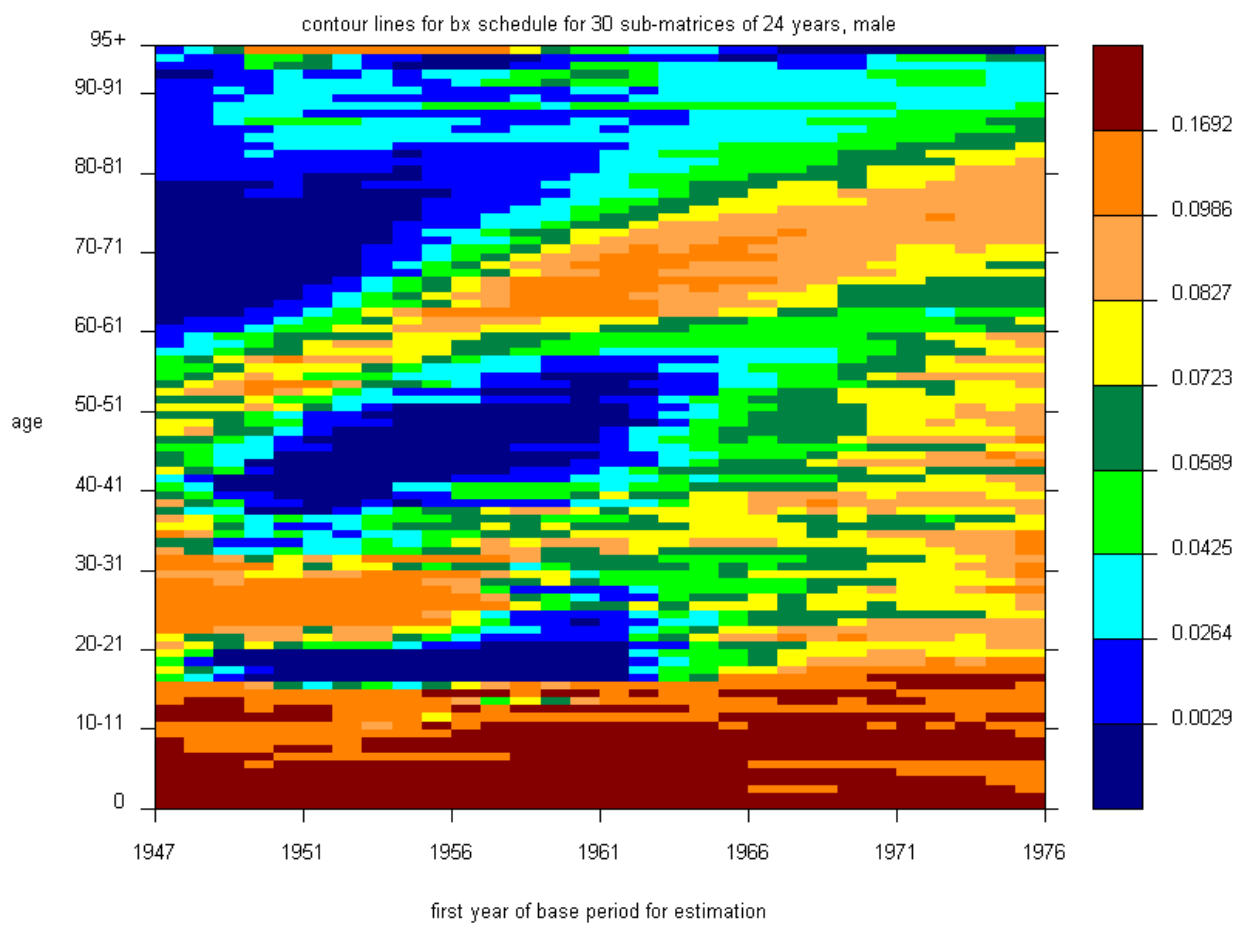




\section{ESTIMATES OF LIFE EXPECTANCY AT BIRTH AND AGE 60 USING THE EXTENDED AND THE ORIGINAL LEE-CARTER METHOD}

To evaluate the goodness of fit of the extended versus the original Lee-Carter method we refer to the difference between the observed and estimated life expectancy at various ages for both methods. Here we present only the results for life expectancy at birth $\left(\mathrm{e}_{0}\right)$ and at age $60\left(\mathrm{e}_{60}\right)$ (further results can be obtained by the authors on request). We have opted to choose the difference between observed and estimated life expectancy as a measure of the goodness of fit since the prediction of life expectancy is central to forecasting mortality rates. The past fit may indicate the best time horizon on which to base future forecasts.

Strong support for the extended Lee-Carter method of modeling mortality rates based on subsamples of the original time series is shown in Figure 5 and Figure 6.

Applying the Lee-Carter method to the whole time series 1947-1999 (cf. Figures 5.a and 5.b), we first over-estimate $e_{0}$ up to the beginning of the 1950s for both males and females and then consistently underestimate it up to 1999 for males and to the late 1980 s for females. From this point onward we slightly over-estimate $e_{0}$ once again. Roughly speaking, the failure to estimate $e_{0}$ correctly is in the range of -0.4 up to +0.4 years. Most interestingly, by applying the Lee-Carter method to subsamples of the original time series the fit essentially improves for all subsamples in the case of females (Figure 5.a). For males the improvement is only visible for the latter subsamples (Figure 5.b). These results may indicate that the structural breaks in the mortality patterns of females and males may require a different time period of subsamples for each gender. This is something that definitely needs to be further explored in future research. As evident in Figure 5.a., the difference between observed and estimated $e_{0}$ can be reduced by almost 0.3 years if one applies the Lee-Carter method to subsamples of the original time series.

An unambiguous improvement in the difference between observed and estimated life expectancy at higher ages, e.g. age 60, is clearly evident for both females and males from the results in Figure 6 . Note that the shape of the bold line, which arises by applying the Lee-Carter method to the whole time series, is almost a mirror image of the corresponding line in Figure 5. The original Lee-Carter method underestimates $e_{60}$ up to the late 1950 s. It then over-estimates $e_{60}$ up to the early to mid-1980s for females (males) and underestimates $e_{60}$ thereafter. It seems that the reduction in mortality at adult and middle ages as evidenced in Figures 3.a. and 3.b for the subsample 1947-1970 may have been attributed to older ages when we applied the Lee-Carter method to the whole time series. Similarly the clear reduction in old age mortality as evidenced for the subsample 1976-1999 in Figure 3.a. and 3.b may have been underestimated when we applied the Lee-Carter method to the whole time series. As evident in Figures 6.a and 6.b, life expectancy at older ages can be modeled more accurately, particularly for the last realization (1976-1999). Hence it is this last subsample on which we will base our forecasts. 
Figure 5.a: Difference in observed and estimated life expectancy at birth for estimates based on the time series 1947-1999 and estimates based on selected subsamples, female

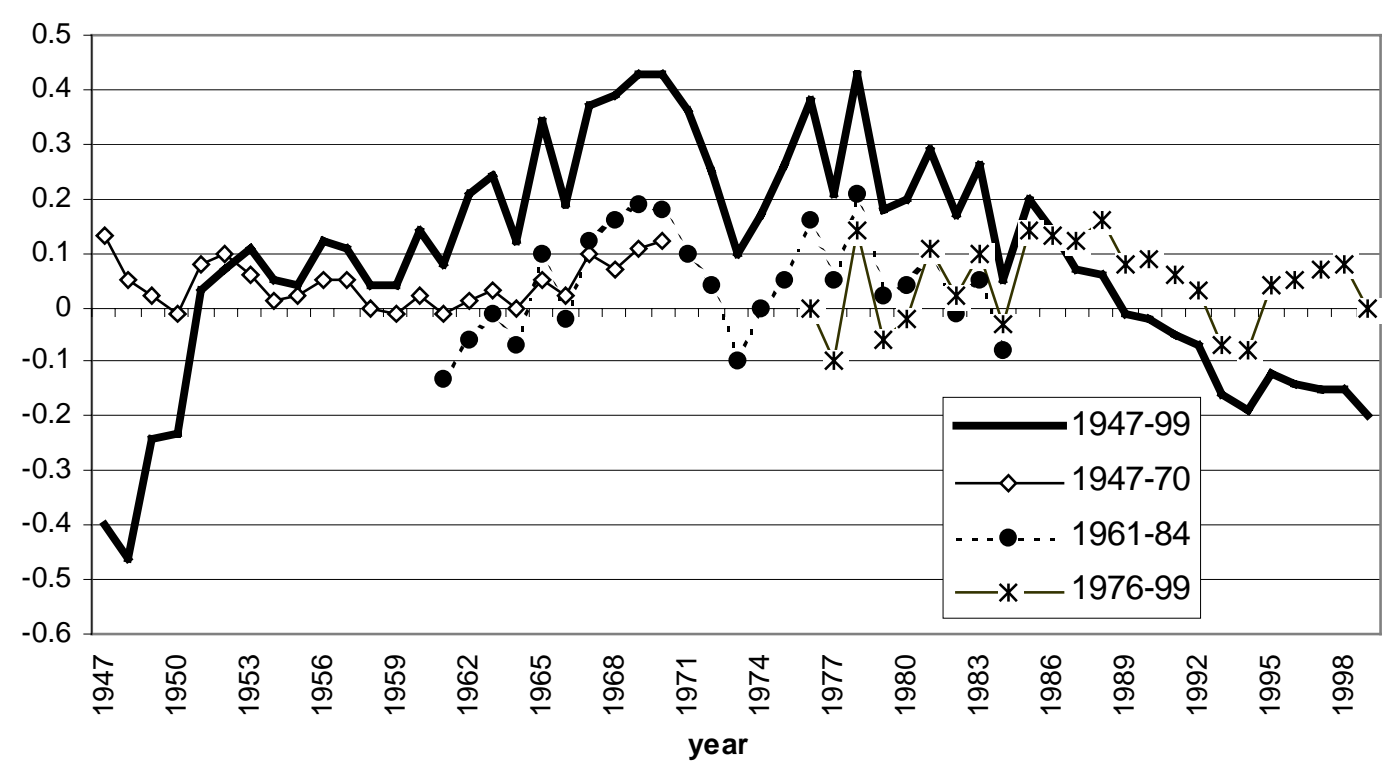

Figure 5.b: Difference in observed and estimated life expectancy at birth for estimates based on the time series 1947-1999 and estimates based on selected subsamples, male

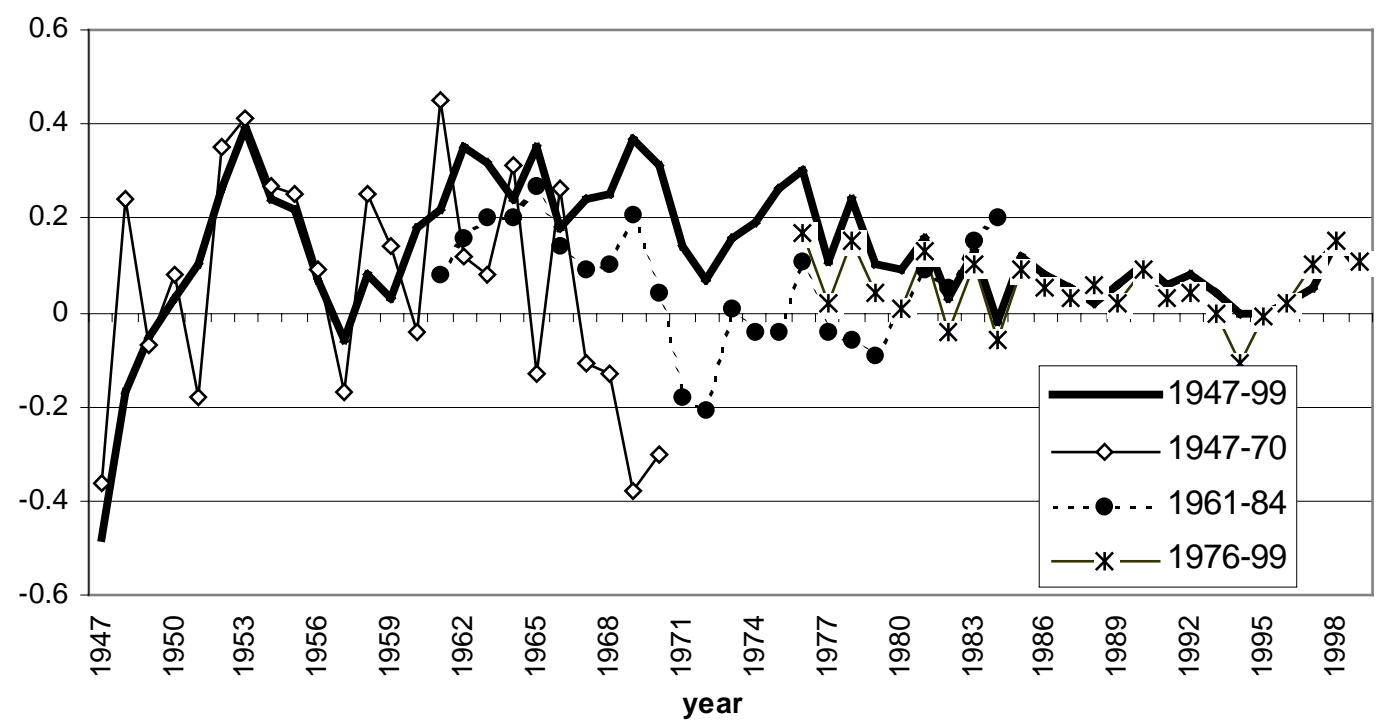


Figure 6.a: Difference in observed and estimated life expectancy at age 60 for estimates based on the time series 1947-1999 and estimates based on selected subsamples, female

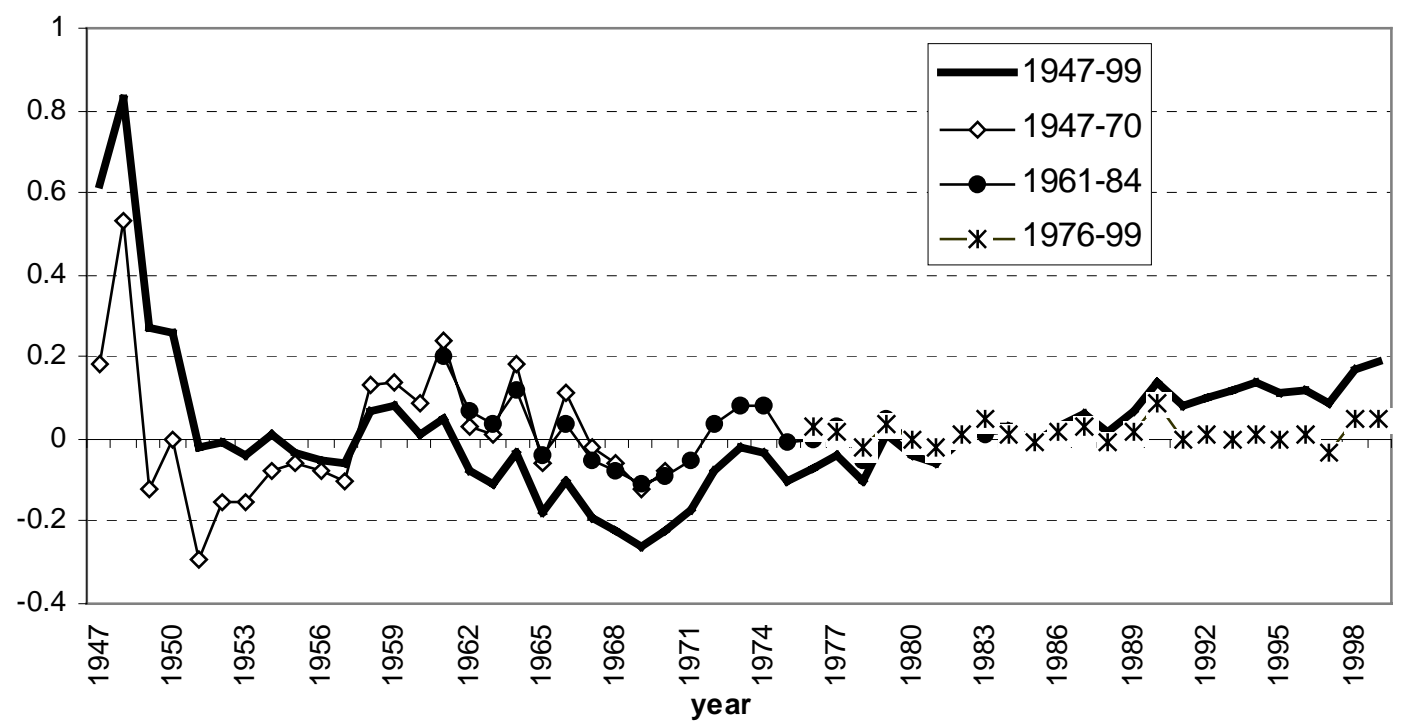

Figure 6.b: Difference in observed and estimated life expectancy at age 60 for estimates based on the time series 1947-1999 and estimates based on selected subsamples, male

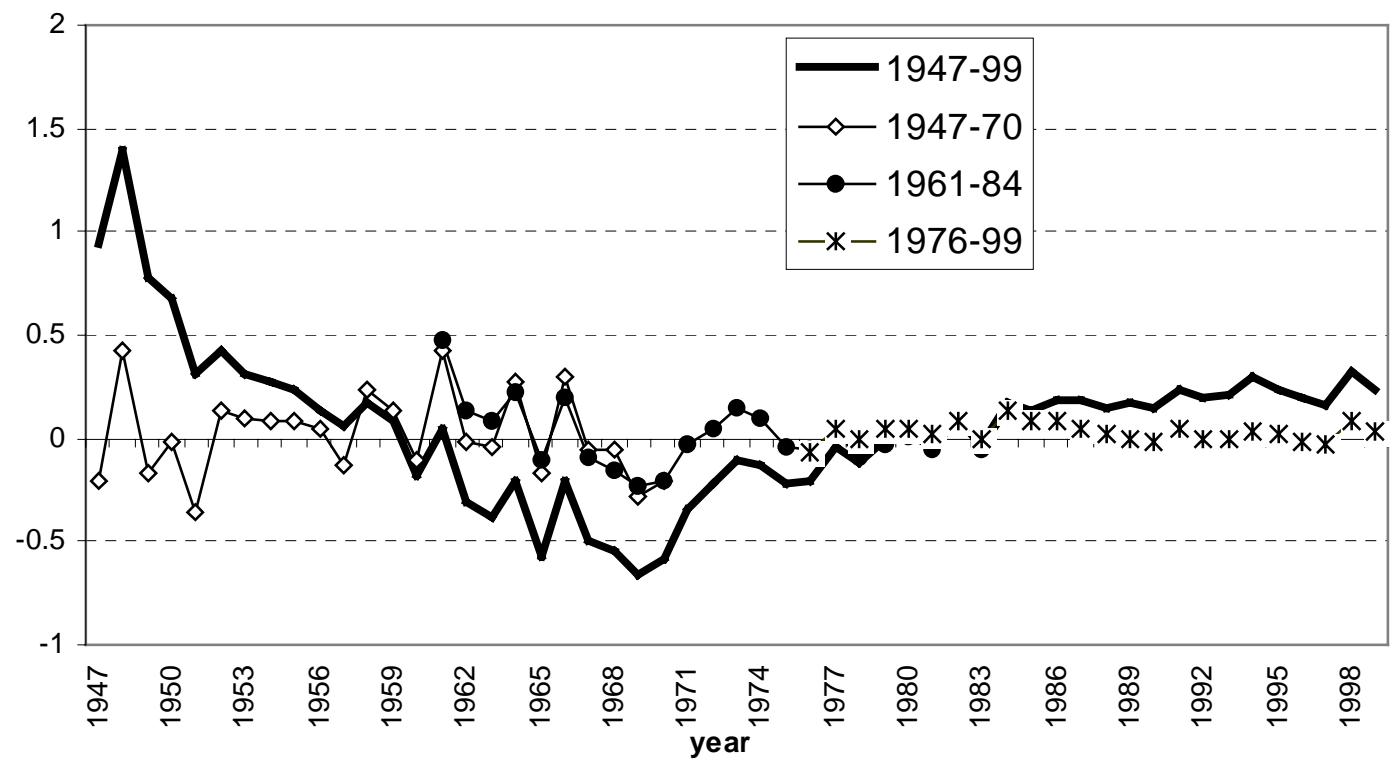




\section{TIME-SERIES MODELING AND FORECASTING}

We employ the Box-Jenkins (1976) techniques for modeling and forecasting the demographic components of the Lee-Carter model. First, we model the entire 53-year series of $k_{t}$ from the original Lee-Carter decomposition with fixed $a_{x} s$ and $b_{x} s$. This estimation provides a benchmark series with which to compare forecasts based on the latest subsample (1976-1999). The estimated time-series model for $k_{t}$ operationally has the formulation:

$$
\mathrm{k}_{\mathrm{t}}=\mathrm{k}_{\mathrm{t}-1}-\text { cnst. }+ \text { noise }
$$

This equation represents a random walk with drift (cnst). Here noise stands for the innovations, which are assumed to be white noise and will not be considered here. This equation is forecasted 51 years ahead to 2050 (similar to Bell 1997, p. 293ff, all forecasts are bias adjusted). These forecasts are recombined with the constants $a_{x} s$ and $b_{x} s$ according to (1) to obtain the forecasted $m_{x, t} s$. The forecasted death rates are used to extract life table functions into the future. The most salient life table function is $e_{0}$, life expectancy at birth (Figures 7a. and 7.b). This process is repeated for a truncated realization of the entire time series ranging from 1976 to 1999; i.e., the latest subsample from the extended Lee-Carter method (Figures 8a. and 8.b).

Figures 7.a and 7.b plot the observed life expectancy at birth up to 1999 together with its forecast (bold line) for females and males respectively. The faded lines indicate the $95 \%$ confidence intervals while the dashed line represents the latest official forecasts from Statistics Austria (Hanika 2000). Note that the official forecast lies within the confidence interval over the entire forecast horizon and is almost identical to the results obtained by the Lee-Carter method for males. According to these figures, life expectancy for females is forecasted to increase from about 81 years in 1999 to 88 years in 2050. Male life expectancy will increase from 75 years to 82 years.

These projected gains in life expectancy may, however, be too conservative, as Figure 8 suggests. Here we plot the forecasts obtained from Figure 7 (bold lines) together with forecasts obtained by applying the Lee-Carter method to the last subsample only, the period from 1976-1999 (dashed lines). In both cases we also add the 95\% confidence interval (solid faded lines and dashed fade lines respectively). For females the difference is only about 1.3 years in 2050, and the forecast based on the subsample 1976-1999 lies within the confidence interval of the forecast based on the whole time series, as can be seen in Figure 8.a. For men, however, the difference in the predicted life expectancy at birth in 2050 in the two methods is about 3.5 years. Moreover the forecast based on the most recent subsample leaves the $95 \%$ confidence interval of the more conservative forecast (which is based on the whole time series) already after the first decade of the $21^{\text {st }}$ century.

We refrain from drawing any definite conclusions about the optimal period on which to base forecasts for future life expectancy at birth. However, our simulations should be taken as evidence that the choice of the base period may not be negligible particularly in the case of the data on Austrian males we use here. 
Figure 7.a: Forecasts of life expectancy at birth based on the time series 1947-1999, female

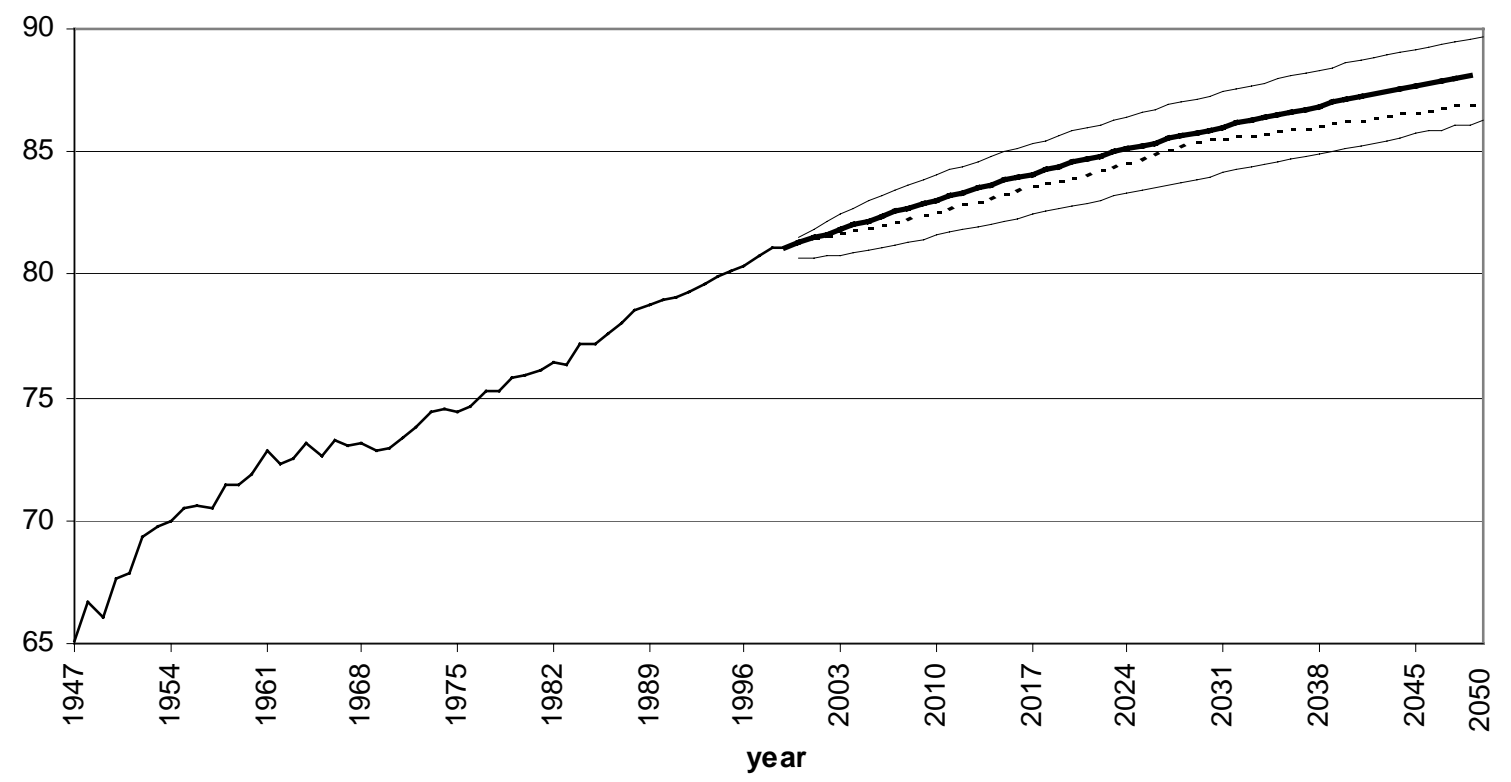

Remark: The dashed line indicates the latest forecast of Statistics Austria and the faded lines indicate the $95 \%$ confidence interval for the Lee-Carter forecast (bold line).

Figure 7.b: Forecasts of life expectancy at birth based on the time series 1947-1999, male

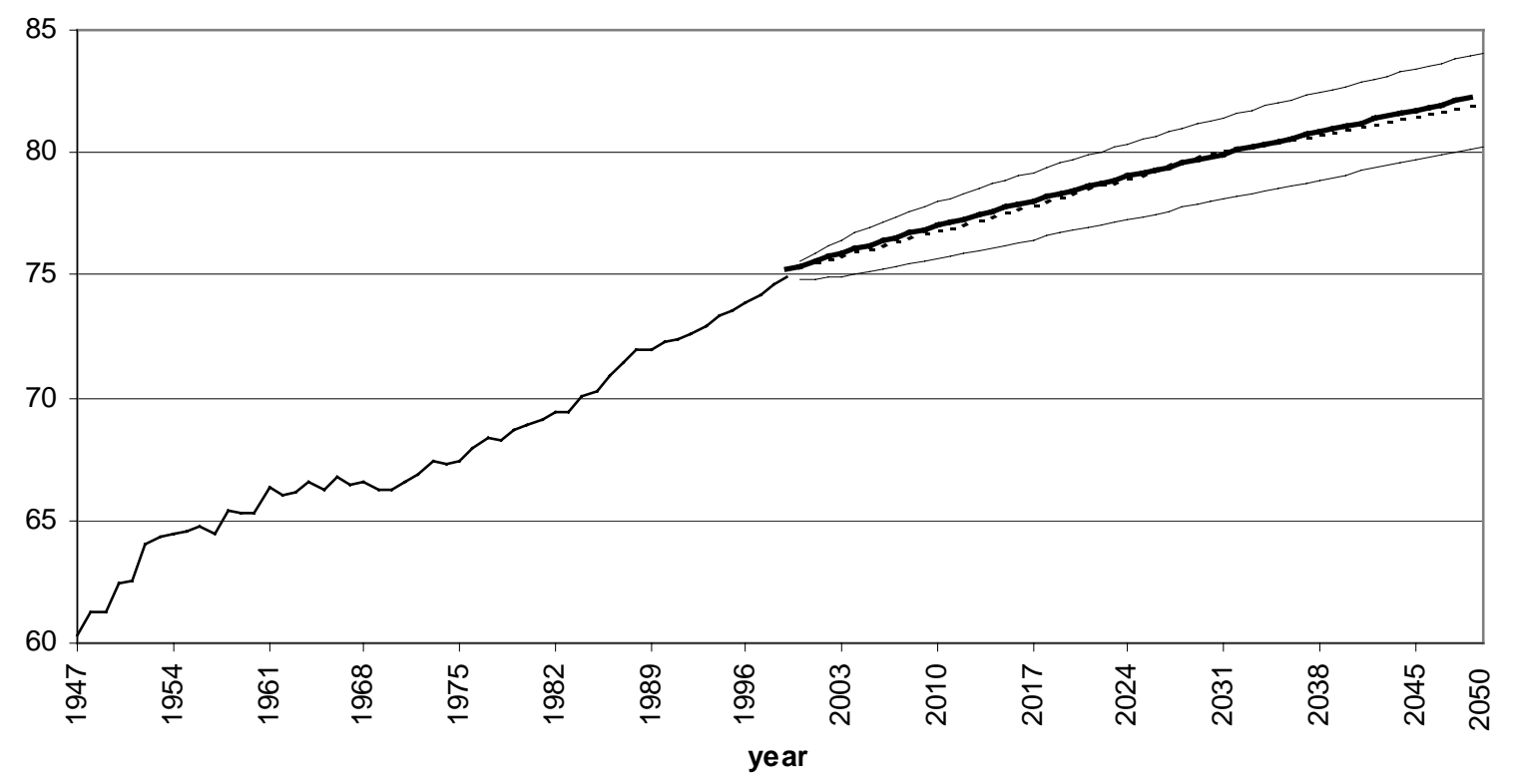

Remark: The dashed line indicates the latest forecast of Statistics Austria and the faded lines indicate the $95 \%$ confidence interval for the Lee-Carter forecast (bold line). 
Figure 8.a: Forecasts of life expectancy at birth based on the time series 1947-1999 and, alternatively, on the time series 1976-1999, female

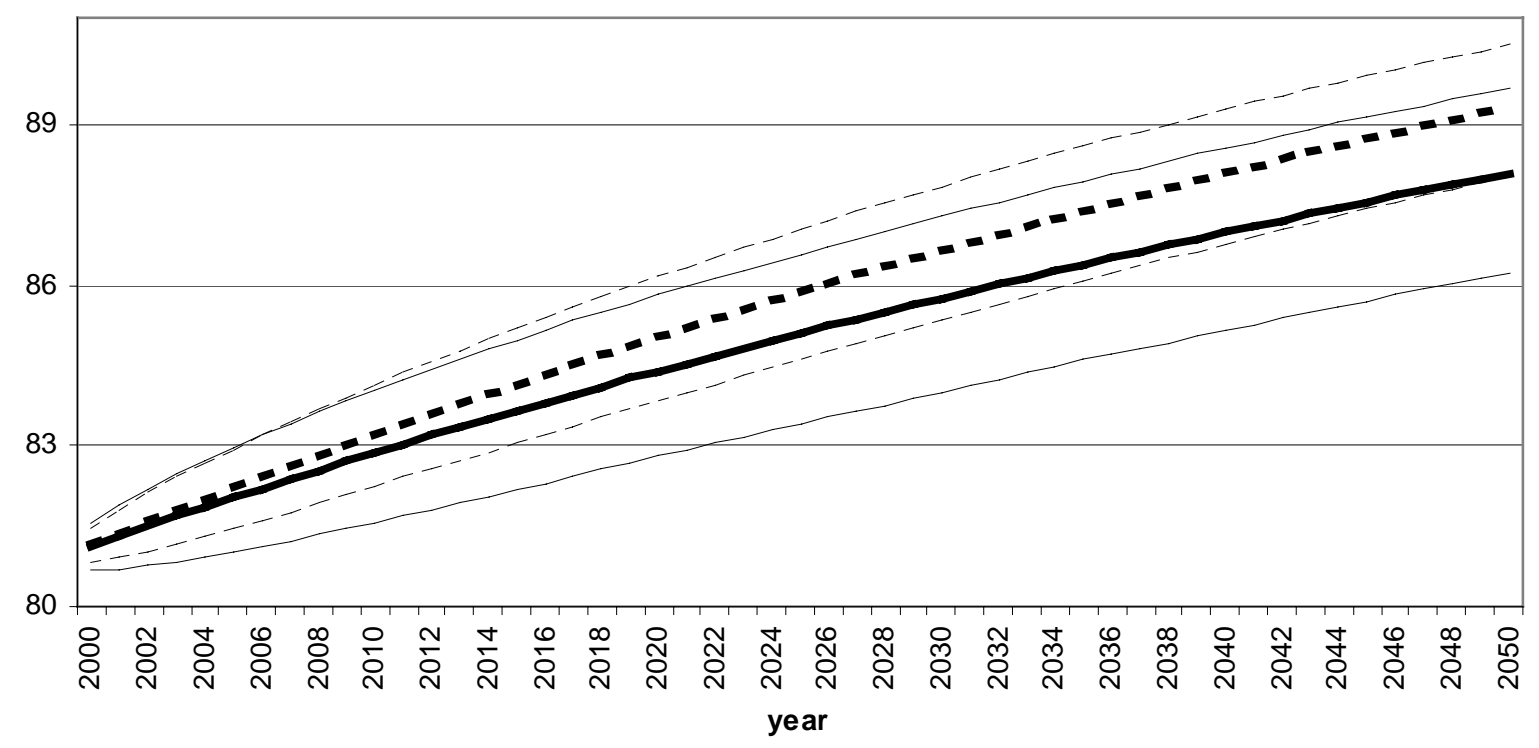

Remark: The solid bold line forecasts based on the time series 1947-99 and the dashed bold line forecasts based on the time series 1976-99 are shown with their corresponding $95 \%$ confidence interval.

Figure 8.b: Forecasts of life expectancy at birth based on the time series 1947-1999 and, alternatively, on the time series 1976-1999, male

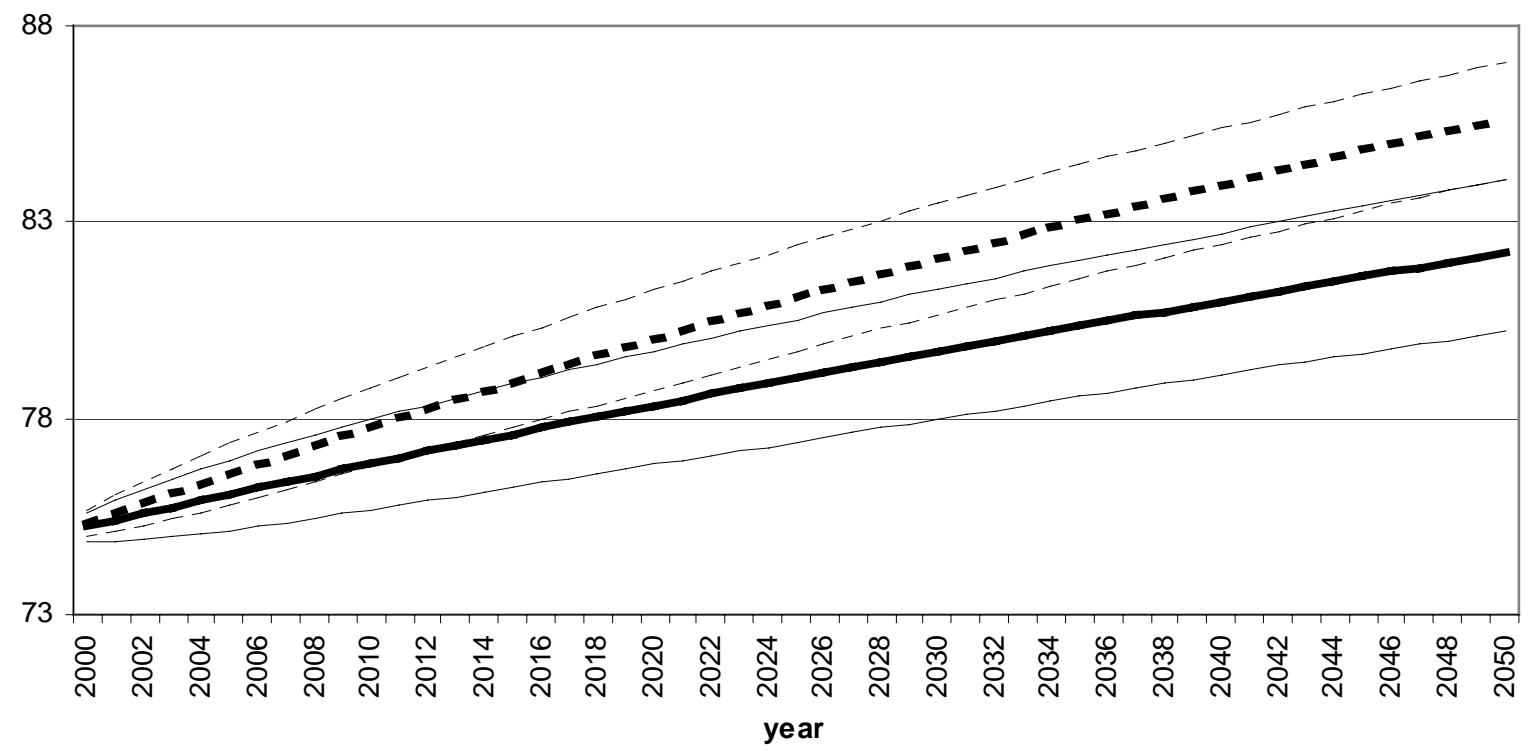

Remark: The solid bold line forecasts based on the time series 1947-99 and the dashed bold line forecasts based on the time series 1976-99 are shown with their corresponding 95\% confidence interval. 


\section{CONCLUSIONS}

We have attempted to show that there is considerably more dynamic behavior in the components of the Lee-Carter model of mortality than is apparent in the single distributions of $a_{x} s, b_{x} s$, and $k_{t} s$. Using the expanded Lee-Carter method, we capture more dimensionality in these components by deriving the matrix arrays $A_{x, s}, B_{x, s}$, and $\mathrm{K}_{\mathrm{t}_{\mathrm{s}}}$ emanating from 3024 -year submatrices in the data. Since a first investigation of these components shows $A_{x, s}$ and $K_{t_{s}}$ to be relatively stable, the focus here was on the components of $B_{x, s}$. Testing various combinations of $A_{x, s}, B_{x, s}$, and $K_{t_{s}}$ showed that the last realization yielded the best estimate of the observed series of $e_{0}$. This test indicates that selectivity is important in discerning the best realization for forecasting mortality. It may be that the last realization is always the best jump-off series for forecasting, though this investigation does not offer proof of this.

This is an initial investigation into the attributes of an expanded Lee-Carter method used for estimating and forecasting mortality. Extensions call for greater explorations of $A_{x, s}$ and $K_{t_{s}}$ matrices. Although they appear to be relatively stable, they may have dynamic characteristics that elucidate mortality patterns more adequately. These techniques need to be applied to other countries - both to countries with mortality histories that are apparently stable and to countries where they appear to be unstable. This should reveal the viability and robustness of this approach. 


\section{BIBLIOGRAPHY}

Andreev, K. F., 1999, Demographic Surfaces: Estimation, Assessment and Presentation, with Application to Danish Mortality, 1835-1995, Ph.D. thesis, University of Southern Denmark

Bell, W.R., 1997, Comparing and assessing time series methods for forecasting agespecific fertility and mortality rates, Journal of Official Statistics 13 (3), 279303.

Box, G.E.P and G.M. Jenkins, 1976, Time Series Analysis: Forecasting and Control (Holden-Day, San Francisco).

Carter, L.R. and R.D. Lee, 1992, Modeling and forecasting U.S. mortality: differentials in life expectancy by sex, in Ahlburg D. and Land K. (eds.) Population Forecasting, a Special Issue of the International Journal of Forecasting Vol.8, no. 3, 393-412.

Hanika, A. (2000) Bevölkerungsvorausschätzung 2000-2050 für Österreich und die Bundesländer, Statistische Nachrichten 12/2000, Statistik Austria, $977 \mathrm{ff}$.

Horiuchi, S. and J.R. Wilmoth, 1995, The aging of mortality decline, paper presented at the Annual Meeting of the Population Association of America, San Francisco, April 6-8, 1995.

Kytir, J. and A. Prskawetz, 1995, Life expectancy at age 60 - epidemiologic scenarios assuming delayed mortality for selected causes of death, European Journal of Population Vol. II, No. 3, 261-273.

Lee, R. and L. Carter, 1992, Modeling and forecasting U.S. mortality, Journal of the American Statistical Association 87(14), 659-671.

Lee, R. and T. Miller, 2000, Assessing the performance of the Lee-Carter approach to modeling and forecasting mortality, paper presented at the Annual Meeting of the Population Association of America, Los Angeles, March 23-25, 2000.

Lee, R. D. and F. Nault, 1993, Modeling and forecasting provincial mortality in Canada, paper presented at the World Congress of the International Union for Scientific Study of Population, Montreal, 1993.

Olshansky, J.S. and B. Ault, 1986, The fourth stage of the epidemiologic transition: the age of delayed degenerative disease, The Milbank Memorial Fund Quarterly 49, 509-538.

Tuljapurkar, S., N. Li and C. Boe, 2000, A universal pattern of mortality decline in G-7 countries, Nature Vol. 405, 789-792.

Wilmoth, J., 1993, Computational methods for fitting and extrapolating the Lee-Carter model of mortality change, Technical Report, Department of Demography, University of California, Berkeley.

Wilmoth, J. R., 1998, Is the pace of Japanese mortality decline converging toward international trends? Population and Development Review 24(3), 593-600. 